\title{
Can skin biopsy differentiate Parkinson disease from multiple system atrophy? And other updates on recent autonomic research
}

\author{
Mitchell G. Miglis ${ }^{1} \cdot$ Srikanth Muppidi ${ }^{2}$
}

Received: 3 July 2020 / Accepted: 4 July 2020 / Published online: 9 July 2020

(c) Springer-Verlag GmbH Germany, part of Springer Nature 2020

Keywords Skin biopsy $\cdot$ Alpha synucleinopathies $\cdot$ Vagal neuropathy $\cdot$ Ultrasound $\cdot$ Multiple system atrophy

Distinguishing multiple system atrophy (MSA) from Parkinson disease (PD) with orthostatic hypotension can be challenging. Even though some features of autonomic cardiovascular reflex testing can be useful, pitfalls still exist and many patients are misdiagnosed, as covered in a prior update [1]. Skin biopsy has recently emerged as a promising technique for in-vivo analysis of phosphorylated alphasynuclein (pSyn) in PD, dementia with Lewy bodies, MSA, and pure autonomic failure as well as the prodromal state of isolated RBD. It is easy to perform, minimally invasive, can be repeated over time, and thus represents an attractive biomarker candidate. In a manuscript recently published in Movement Disorders, Donadio and colleagues discuss how dermal immunofluorescence analysis may offer some additional clues to help clinicians with such challenging cases [2].

The authors recruited 50 patients with Parkinsonism and chronic neurogenic orthostatic hypotension (nOH): 25 with probable MSA-P and 25 with PD. Although most patients underwent autonomic cardiovascular testing, including head-up tilt, $25 \%$ of patients only had an active standing testing for the diagnosis of nOH. Skin biopsy samples were taken from the cervical (C7) paraspinal area, thigh $(15 \mathrm{~cm}$ above the patella) and distal leg $(10 \mathrm{~cm}$ above the lateral malleolus). A second skin biopsy was taken $3-4 \mathrm{~cm}$ from the first site to confirm accuracy and increase sensitivity. Twenty-three age-matched healthy subjects without neurological deficits served as controls. All patients completed several ratings scales for autonomic symptoms including the

Srikanth Muppidi

muppidis@stanford.edu

Stanford Medical Center, Palo Alto, CA, USA

2 Stanford Neurosciences Health Center, 213 Quarry Road, 2nd Floor, Palo Alto, CA 94304, USA
COMPASS-31, the UMSARS-1, and the Non-Motor Symptoms Scale.

Abnormal pSyn deposits were found in 18 out of 25 (72\%) patients with MSA, with the distal leg being the site with highest positivity (100\%, compared to $78 \%$ at the thigh and $67 \%$ at C7). Donadio and other groups have reported a patchy dermal distribution of pSyn across synucleinopathies, thus supporting the decision to biopsy multiple sites to increase yield. The authors found that, when only one site was analyzed, just $40 \%$ of the skin samples from patients with MSA showed pSyn staining. The percentage increased to $72 \%$ when the second biopsy from each site was added. By comparison, pSyn was present in $100 \%$ of the samples from patients with $\mathrm{PD}$ and $\mathrm{OH}$.

The second finding of interest was the difference in pSyn distribution in samples from patients with MSA compared to those of patients with PD and $\mathrm{OH}$. All patients with MSA had pSyn deposits in somatic nerves of the subepidermal plexi, however only $28 \%$ demonstrated deposits in the autonomic nerves. The converse finding was seen in patients with $\mathrm{PD}$ and $\mathrm{OH}$, with the majority of pSyn deposits found in typical dermal autonomic locations, i.e., sweat glands, pilomotor and vasomotor structures, but not in the epidermal somatic nerves themselves. In addition, the distribution of pSyn was seen in a less rostro-caudal gradient than seen in the MSA group, with more even distribution across sites. Finally, the patients with MSA demonstrated a more pronounced decrease in epidermal nerve fiber density across all sites, although the difference was not significant. This finding warrants further investigation, especially in relation to the underrecognized symptom of neuropathic pain in MSA.

The burden of pSyn did not correlate with age, disease duration, L-dopa dose, severity of $\mathrm{nOH}$, or the results of autonomic rating scales. The authors found that the best post-hoc accuracy was found when pSyn positivity was detected in somatic nerves or when somatic and autonomic 
nerves were both negative [sensitivity $100 \%, 95 \%$ confidence interval (CI), 98-100; specificity $84 \%, 95 \%$ CI, 82-86]. Maximizing specificity, the best accuracy was found when the autonomic nerves were negative with any result for somatic nerve structures (sensitivity $88 \%, 95 \% \mathrm{CI}$, $86-90$; specificity $100 \%, 95 \%$ CI, 98-100). This result supports a different distribution of pSyn in the cutaneous nerves in MSA-P (mostly affecting somatic nerves) compared to $\mathrm{PD}+\mathrm{OH}$ (mostly affecting autonomic nerves), with a diagnostic indication in favor of MSA-P when both nerves were negative.

Additional limitations of this study include the lack of neuropathologic confirmation, a shortcoming for most MSA studies, as well as limited reported autonomic testing data. It is also unclear why the authors chose to report a 3-min head-up tilt result, as compared to the more standard 10-min interval. Nonetheless, this data represent another landmark step forward by the cutaneous laboratory of the Bologna investigators, and further support the promise of skin biopsy as both a diagnostic and prognostic tool for the future.

\section{Vagus nerve recording: the future is almost here}

In a typical autonomic laboratory, efferent vagal activity can be indirectly measured with heart rate variability analysis and gastrointestinal motility studies. Assessing the function and structure of sensory and motor peripheral nerves is routinely done with nerve conduction studies and peripheral nerve biopsy. However, stimulation of autonomic nerves is not routinely performed, and there is no easy way to quantify the structure and density of the autonomic innervation. These limitations highlight the need for additional methods of physiological and anatomical measures of autonomic nerves. Currently, post-ganglionic sympathetic activity can be measured directly from the peripheral nerve with needle microneurography, however a similar technique had never been used in humans with a direct recording from the vagus nerve, even though vagus nerve stimulation is currently used as a therapeutic approach in multiple diseases including epilepsy and depression.

In a recent article published in the Journal of Physiology, Ottaviani, Macefield and colleagues [3] attempted to directly measure activity from the vagus nerve. They used an intraneural tungsten electrode and obtained direct nerve recordings from the right and left vagus nerves in three patients. The recording needle was inserted with ultrasound guidance in the neck, posterior to the sternocleidomastoid muscle. The authors recorded firing rates from these neurons to assess end-organ innervation, finding that the neuronal firing pattern was inversely correlated with heart rate function and these were likely the efferent preganglionic vagal fibers to the sinoatrial node. Additionally, they identified fibers which were likely afferent fibers from the upper airway and efferent motor fibers to the larynx. They were unable to isolate efferent vagal fibers innervating the gastrointestinal tract, as there was no clear and direct gastrointestinal motility measurement during vagal nerve recording.

This first-in-human study, which is novel in many ways and proves the feasibility of this technique, will need to be replicated extensively before we can clarify all the components of the vagus nerve and correlate its firing patterns with the multiple autonomic functions it performs. As the authors note, they will likely need to replicate these findings in a larger group along with pharmacological and dynamic studies that alter the function of the vagus nerve.

This above technique is exciting news but is unlikely to be used in clinical practice due to the complexity and risk of such an invasive procedure. An alternative to assess the structural integrity of the nerves is neuromuscular ultrasound. In a recent publication in the Journal of Neuroimaging, Niu and colleagues [4] report their experience using ultrasound to quantify the structure of the vagus nerve measurement in patients with chronic inflammatory demyelinating polyneuropathy (CIDP) and Charcot-Marie Tooth disease type 1A (CMT1A). This works expands on previous work by multiple groups on the feasibility of obtaining vagus nerve anatomical measures in patients with known neuropathies. The authors recruited 43 patients with CIDP, 8 with CMT1A, and 105 healthy controls. The cross-sectional areas of the vagus, median, and ulnar nerves were measured bilaterally and these cross-sectional areas were followed longitudinally in the case of the CIDP patients. Cross-sectional areas were higher in CIDP and CMT1A compared to healthy controls. The cross-sectional area of the vagus nerve positively correlated with the maximum crosssectional area of median/ulnar nerve in CIDP and CMT1A. Furthermore, the change in vagus nerve cross-sectional area positively correlated with the change in mean median/ulnar nerve cross-sectional area in CIDP after treatment with corticosteroids. These findings suggest that the cross-sectional area of the vagus can be reliably measured in a diverse group of patients. Interestingly, autonomic neuropathy, and specifically vagal neuropathy, is not considered a primary pathology in patients with CIDP or CMT1A and on previous analysis patients did not have abnormalities on autonomic testing of vagal function.

Whether changes on ultrasound in this study reflect diffuse and nonspecific nerve involvement in CMT1A or CIDP or are a measure of subclinical autonomic nerve involvement is unclear. Nonetheless, ultrasound offers a reliable anatomical measure of an autonomic nerve, though the value of this metric in research and clinical practice is yet to be determined. 
Funding None.

\section{Compliance with ethical standards}

Conflict of interest None.

\section{References}

1. Miglis MG, Muppidi S (2019) The microbiome in autonomic medicine and other updates in recent autonomic research. Clin Auton Res 29:361-362
2. Donadio V, Incensi A, Rizzo G et al (2020) Skin biopsy may help to distinguish multiple system Atrophy-Parkinsonism from Parkinson's Disease with orthostatic hypotension. Mov Disord 16:8. https://doi.org/10.1002/mds.28126

3. Ottaviani MM, Wright L, Dawood T, Macefield VG (2020) Invivo recordings from the human vagus nerve using ultrasoundguided microneurography. J Physiol 28:77

4. Niu J, Zhang L, Ding Q et al (2020) Vagus nerve ultrasound in chronic inflammatory demyelinating polyradiculoneuropathy and Charcot-Marie-tooth disease type 1A. J Neuroimaging 12:7-47 